\title{
Matrix changes induced by transglutaminase 2 lead to inhibition of angiogenesis and tumor growth
}

\author{
RA Jones ${ }^{1,5}$, P Kotsakis ${ }^{1,5}$, TS Johnson ${ }^{2}$, DYS Chau ${ }^{1,4}$, S Ali ${ }^{1}$, \\ G Melino ${ }^{3}$ and M Griffin ${ }^{\star, 4}$ \\ 1 School of Biomedical and Natural Sciences, Nottingham Trent University, \\ Nottingham NG11 8NS, UK \\ 2 Sheffield Kidney Institute, Northern General Hospital, Sheffield \\ S5 7AU, UK \\ 3 Biochemistry Laboratory IDI-IRCCS, c/o Department of Experimental \\ Medicine, University of Rome, 'Tor Vergata', 00133 Rome, Italy \\ ${ }^{4}$ School of Life \& Health Sciences, Aston University, Birmingham, UK \\ 5 These authors contributed equally to this work \\ * Corresponding author: M Griffin, School of Life and Health Sciences, Aston \\ University, Aston Triangle, Birmingham B4 7ET, UK. Tel: + 44121 2043942; \\ Fax: + 44121 3336350; E-mail: M.Griffin@aston.ac.uk
}

Received 12.4.05; revised 24.8.05; accepted 22.9.05; published online 18.11 .05 Edited by A Finazzi-Agrò

\begin{abstract}
Administration of active TG2 to two different in vitro angiogenesis assays resulted in the accumulation of a complex extracellular matrix (ECM) leading to the suppression of endothelial tube formation without causing cell death. Matrix accumulation was accompanied by a decreased rate of ECM turnover, with increased resistance to matrix metalloproteinase-1. Intratumor injection of TG2 into mice bearing CT26 colon carcinoma tumors demonstrated a reduction in tumor growth, and in some cases tumor regression. In TG2 knockout mice, tumor progression was increased and survival rate reduced compared to wild-type mice. In wild-type mice, an increased presence of TG2 was detectable in the host tissue around the tumor. Analysis of CT26 tumors injected with TG2 revealed fibrotic-like tissue containing increased collagen, TG2-mediated crosslink and reduced organized vasculature. TG2-mediated modulation of cell behavior via changes in the ECM may provide a new approach to solid tumor therapy.

Cell Death and Differentiation (2006) 13, 1442-1453.

doi:10.1038/sj.cdd.4401816; published online 18 November 2005
\end{abstract}

Keywords: transglutaminase 2; inhibition; angiogenesis; solid tumor; extracellular matrix

Abbreviations: TG2, transglutaminase 2; ECM, extracellular matrix; MMP, matrix metalloproteinase; HUVEC, human umbilical large vein endothelial cells; FCS, fetal calf serum; BSA, bovine serum albumin; vWF, von Willebrand factor; HRP, horseradish peroxidase; LDH, lactate dehydrogenase; TRITC, tetramethyl rhodamine isothiocyanate; FITC, fluorescein isothiocyanate; CBZ, benzyloxycarbonyl; EDTA, ethylene diamine tetraacetic acid; UPARAP, urokinase plasminogen activator receptor-associated protein

\section{Introduction}

The growth and development of new tissue relies on the generation of a new blood supply. Angiogenesis, the process of new blood vessel formation, is fundamental to wound healing, embryonic development, and pathological conditions such as solid tumor growth and rheumatoid arthritis. ${ }^{1}$ Angiogenesis is a complex process involving many growth factors, enzymes, different cell types, and changes to the extracellular matrix (ECM). Importantly, the control of inappropriate angiogenesis in diseases such as cancer has led to the development of novel antiangiogenic agents that interfere with endothelial cell proliferation, migration, and invasion. ${ }^{2}$ Numerous strategies have been adopted, such as gene therapy, monoclonal antibodies, and peptides. ${ }^{3}$ However, technical challenges remain both with the delivery of these agents and in their reliability, particularly with respect to their use as antineoplastic agents. ${ }^{4}$

New blood vessel formation is dependent on changes in the behavioral characteristics of endothelial cells: in particular their proliferation, migration, and differentiation into tubular structures, which is influenced by changes in the ECM. Endothelial cells are a rich source of tissue transglutaminase (TG2), ${ }^{5}$ a multifunctional enzyme that catalyzes the multimerization of proteins by generating $\varepsilon(\gamma$-glutamyl)lysine isopeptide bonds. ${ }^{6}$ Many ECM proteins are known substrates of the enzyme, ${ }^{7}$ and the crosslinking of these proteins by endothelial cell TG2 is thought to play a role in the stabilization of the basement membrane. ${ }^{8}$ However, in a number of different tissues, inappropriate crosslinking of the ECM by TG2 following continuous tissue insult can lead to ECM accumulation and fibrosis. ${ }^{9,10}$ Cell surface TG2 is also thought to be involved in cell migration, ${ }^{11}$ and cell adhesion, ${ }^{12}$ by a mechanism that is independent of its transamidating activity. ${ }^{13}$ While some of these proposed functions of TG2 are similar to the critical steps involved in angiogenesis, the role of TG2 in this process is poorly understood. TG2 knockout mice do not present vascular abnormalities ${ }^{14}$ and it has been reported that TG2 is downregulated in endothelial cells undergoing capillary morphogenesis. ${ }^{15}$ The latter observation fits with the proposed extracellular role of the enzyme in ECM deposition and stabilization, since angiogenesis requires localized destabilization of the matrix brought about by its increased turnover. ${ }^{16}$ However, conflicting in vivo studies have proposed that the enzyme both stimulates and inhibits angiogenesis. ${ }^{17,18}$

As a consequence of these different findings, we first investigated whether TG2, via its ability to cause changes in the ECM , plays a regulatory role in capillary tube development. Additionally, we investigated whether this regulatory effect on cell behavior could be exploited in the control of tumor growth, a process that is also influenced by changes in the ECM. Using in vitro angiogenesis assays, we demonstrate that application of exogenous TG2 blocks angiogenesis in a dose-dependent manner without causing cell death, by a 
mechanism that involves increased accumulation of ECM proteins. Extension of these studies in vivo using two different tumor models demonstrated that the increased presence of TG2 in the tumor environment leads to delayed tumor growth and increased animal survival rates by a mechanism that is dependent on changes in the ECM. We propose that TG2 could be a novel and useful tool for modulating cell behavior when used to induce changes in the stability of the ECM leading to fibrosis, resulting in a reduction in angiogenesis and subsequently inhibition of tumor growth.

\section{Results}

\section{TG2 activity and expression in human in vitro angiogenesis}

Since endothelial cells are a rich source of TG2, we initially examined the distribution of the enzyme antigen and the localization of in situ enzyme activity in the human in vitro coculture model of angiogenesis (Figure 1a). Immunoperoxidase staining for TG2 antigen revealed an abundance of enzyme in capillary networks in a pattern that resembled staining for von Willebrand factor (vWF) (Figure 1b). By incubating cultures with the competitive primary amine TG substrates biotin (Figure 1c) or fluorescein cadaverine (Figure 1d), which become incorporated into protein-bound glutamine by TG2, it is possible to detect the presence of in situ TG activity. Biotin cadaverine-treated cultures indicated that most of the endogenous enzyme activity was located in the capillary networks, and examination of fluorescein cadaverine-treated cultures by high-power confocal microscopy demonstrated that this activity was primarily located at the endothelial cell surface, in cellular debris, and in the ECM surrounding the endothelial cells.

Inhibition of TG2 activity using the active-site-directed irreversible inhibitors of the enzyme NTU281 (Figure 1e) or NTU 283 (data not shown) at concentrations up to $1 \mathrm{mM}$ had no significant effect on tubular development. Confirmation that these inhibitors were blocking transglutaminase activity was provided by their ability to inhibit the incorporation of fluorescein cadaverine into ECM proteins (Figure 1f).

\section{Effect of exogenous TG2 treatment on endothelial tube formation}

As a means of increasing the extracellular presence of TG2, active TG2 $(50 \mu \mathrm{g} / \mathrm{ml}, 50 \mathrm{U} / \mathrm{ml})$ was added to the in vitro coculture model of angiogenesis at different stages of its development, which resulted in a dose-dependent inhibition of tube formation compared to untreated cultures (Figure 2a). Suppression of capillary development was seen after two TG2 treatments and optimal inhibition of angiogenesis was achieved following four applications of enzyme. TG2-treated vessels appeared highly truncated and exhibited reduced capillary branching. Quantification of vWF staining by image analysis in TG2-treated and untreated angiogenesis cultures indicated that capillary length, number of vessels, number of vessel-branching points, and percentage area covered by the endothelial tubules were all significantly reduced in a dose-dependent manner in TG2-treated cultures (Figure 2b).
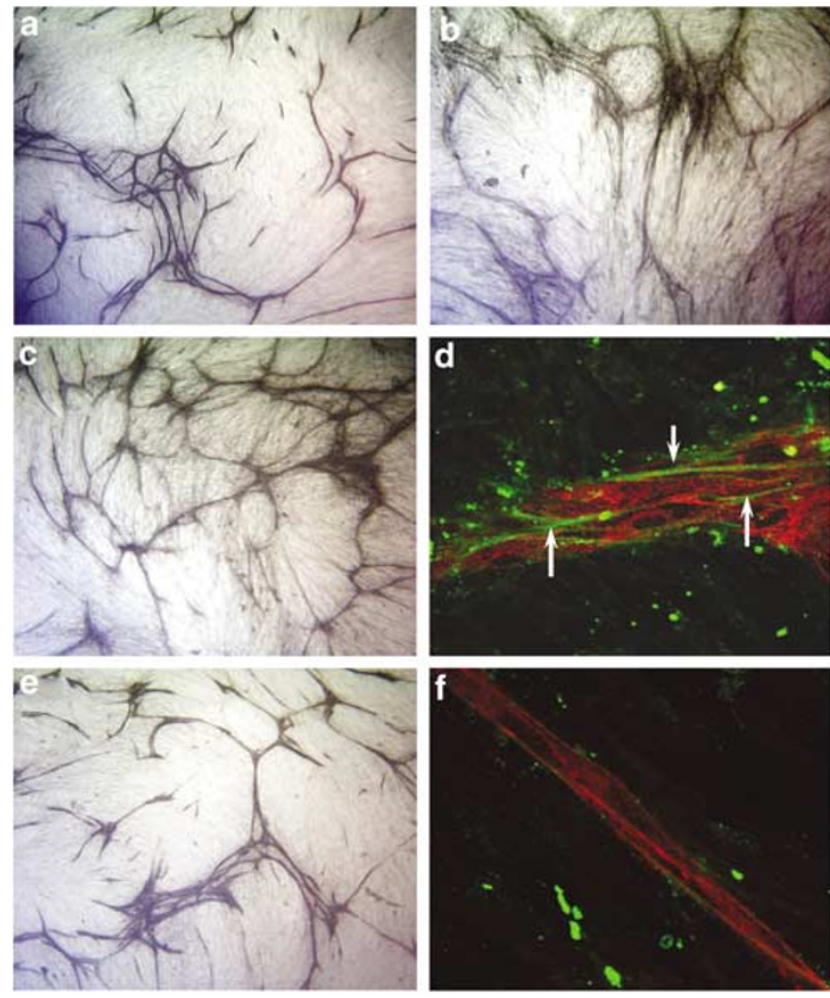

Figure 1 Endogenous TG2 expression and activity in a human angiogenesis cell model. (a) Demonstration of the capillary networks formed in the HUVEC/ fibroblast coculture system. Cells are cultured for 11 days and HUVEC stained with an anti-vWF antibody. (b) TG2 immunohistochemistry in an angiogenesis cell model. (c) Endogenous in situ TG2 activity visualized by biotin cadaverine incorporation. In (d), endogenous in situ TG2 activity (fluorescein cadaverine incorporation - green) is shown with HUVEC counterstain (vWF - red) by confocal microscopy. TG2 activity is predominantly targeted to the ECM surrounding endothelial cells (arrows). (e) Inhibition of endogenous TG2 activity with $1 \mathrm{mM}$ NTU281 has little effect on capillary development. (f) TG2 inhibitor treatment at $1 \mathrm{mM}$ prevents fluorescein cadaverine incorporation (green) in the capillary ECM, HUVEC are red. Original magnification: (a, b, $\mathbf{c}$, and $\mathbf{e})$ are $\times 8$, (d and f) are $\times 40$

To establish whether transamidating activity was required to inhibit angiogenesis, cultures were also treated in parallel with TG2, which had been inactivated by preincubation with the active site-directed inhibitor NTU283 (500 $\mu \mathrm{M})$. Inhibition of the transamidating activity of the applied TG2 reversed the enzymes' antiangiogenic properties (Figure $2 a$ and b). In order to confirm the inhibition observed with the coculture angiogenesis model, TG2 was also added in a similar manner (four applications of enzyme, $50 \mu \mathrm{g} / \mathrm{ml}, 50 \mathrm{U} / \mathrm{ml}$ ) to an aorta ring model of angiogenesis. Following four daily additions of exogenous TG2 to a 10-day incubation culture (Figure 2c) microvessel formation was completely inhibited (Figure 2c).

\section{TG2-mediated inhibition of angiogenesis is not caused by induction of cell death}

After addition of the enzyme to the coculture model, few dead cells were detected regardless of the TG2 treatment (Figure 3a). The toxicity of applied TG2 to single-cell populations of human umbilical large vein endothelial cells 
(HUVEC) and C378 cultures, which account for the core cell population of angiogenesis cocultures, was also investigated. No effect of TG2 treatment on cell viability of either cell type was observed when cell density was measured using the XTT assay (Figure $3 c$ ), confirming the data shown in Figure 3a. Interestingly, lactate dehydrogenase (LDH) release, an indicator of cell permeability, indicated decreased cell permeability in the TG2-treated cells (Figure 3b).

\section{Localization of exogenous TG2 activity in enzyme-treated cultures}

In untreated cells, endogenous in situ TG activity was observed in the ECM and was particularly concentrated in anastomosing endothelial capillaries (Figure 4a). Following two applications of TG2, the activity of the exogenous enzyme was seen in thick fibrillar structures that surrounded endo-
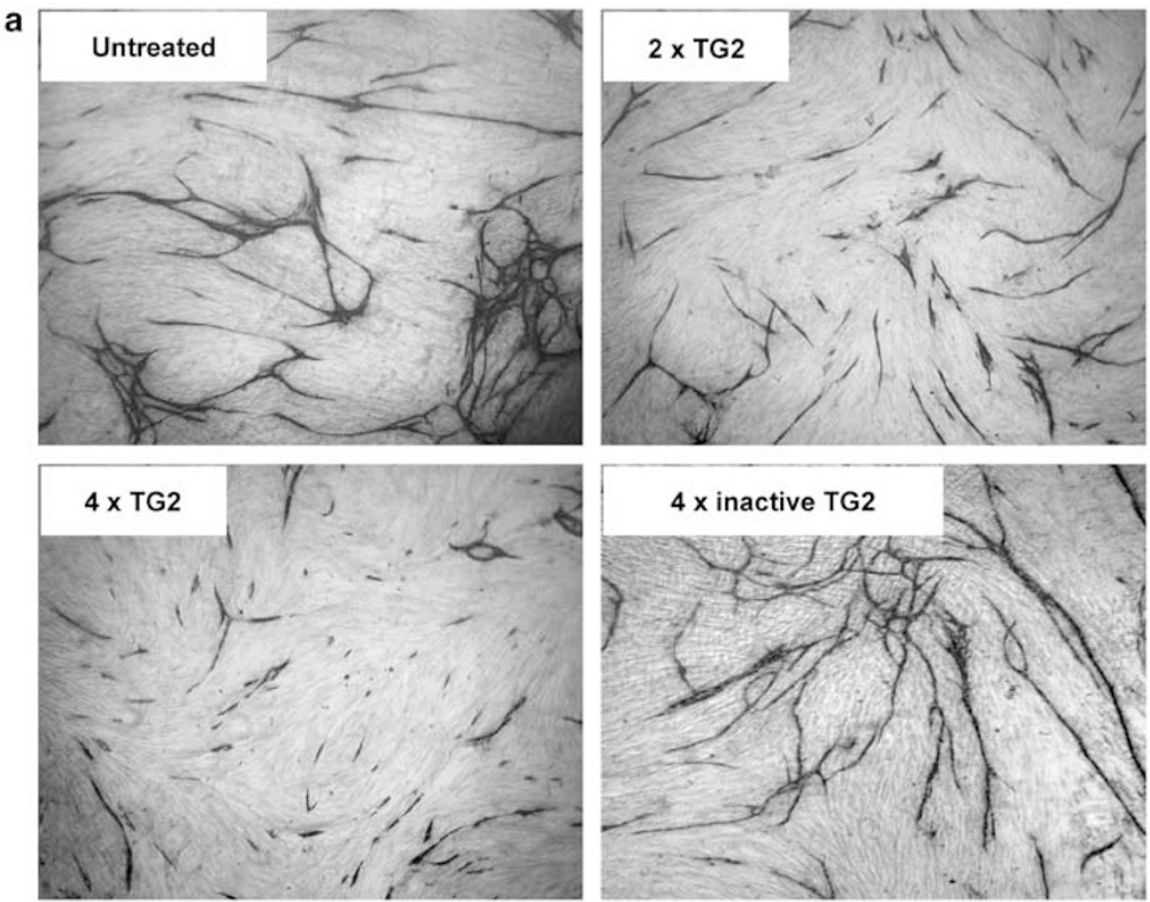

b

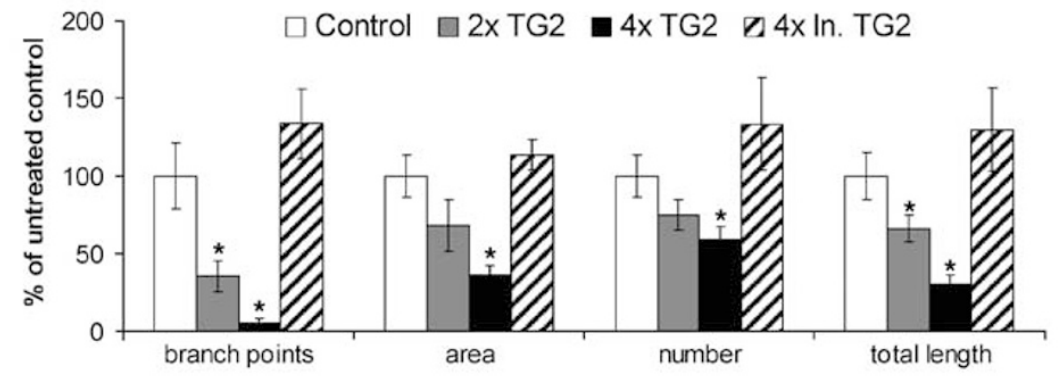

c
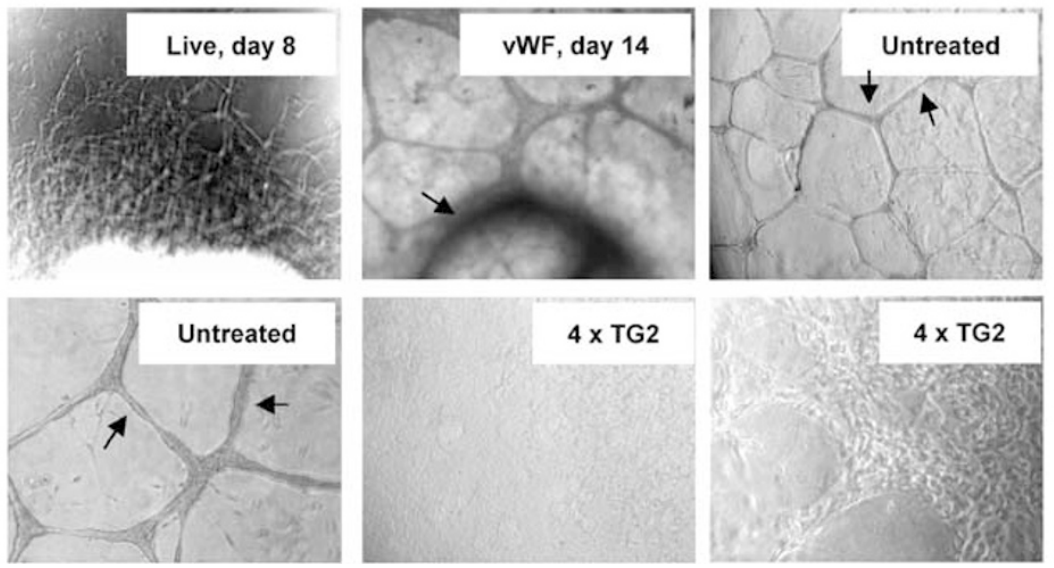
thelial capillaries and extended into the surrounding ECM. Upon four administrations, the ECM-associated TG activity appeared to be considerably increased and the matrix-associated incorporation appeared much denser with finer branching random structures, while endothelial cell vasculatures were largely absent. By observing a field from top to bottom containing a small endothelial cell population at high magnification, TG2 activity measured in situ could be seen to be present in fibrillar structures encasing a capillary.

\section{Progressive TG2 treatment of angiogenesis cultures leads to polymerization of proteins}

Treatment of the coculture model with TG2 revealed selfincorporation of the enzyme into high-molecular-weight polymers when cellular proteins were separated by SDSPAGE, Western blotted, and then immunoprobed with the
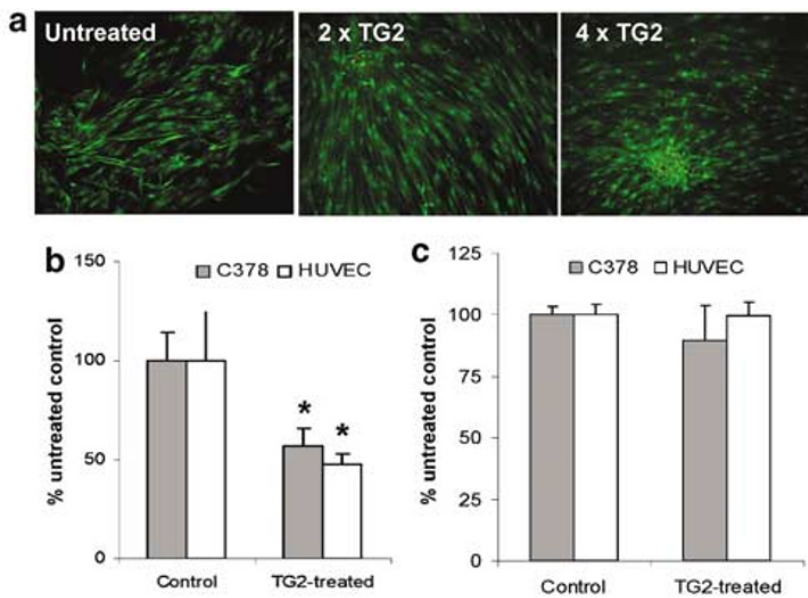

Figure 3 TG2 treatment is not cytotoxic to angiogenesis coculture cell model, HUVEC or human dermal fibroblast cells. (a) Cytotoxicity visualized in situ by LIVE/DEAD differential viability stain following 11 days in culture and TG2 treatment. Live cells with intact membranes are green, dead cells with leaky membranes are red. The left shows untreated cells, the middle shows cultures receiving two TG2 treatments on days 2 and 5 and the right shows cultures treated four times with TG2 on days 2, 5, 7, and 10. (b) shows LDH release in C378 dermal fibroblasts (shaded bars) and HUVEC (open bars) following treatment with TG2 $(50 \mu \mathrm{g} / \mathrm{ml}, 50 \mathrm{U}$ per treatment). (c) shows the effect of TG2 on cell proliferation using XTT in C378 dermal fibroblasts (shaded bars) and HUVEC (open bars) following treatment with TG2 $(50 \mu \mathrm{g} / \mathrm{ml}, 100 \mathrm{U}$ per treatment). Bars in $(\mathbf{b}$ and $\mathbf{c})$ show \pm S.E. *Significant difference compared to control $(P<0.05)$
Cub7402 MAb specific for TG2 (Figure 4b). This polymerization increased with repeated dosing of the enzyme. Cultures treated with four repeated applications of TG2 revealed the enzyme to be present in both high-molecular-weight aggregates and numerous degradation fragments. Immunoprobing of blots for fibronectin indicated this to be present in nonreducible high-molecular-weight aggregates, which were unable to penetrate the resolving and stacking gels in a dosedependant manner. Elevated $\varepsilon(\gamma$-glutamyl)lysine crosslinks were also revealed in immunoblots with increased TG2 treatment. Cultures treated with one or two doses of TG2 demonstrated increased presence of the $\varepsilon(\gamma$-glutamyl)lysinecontaining proteins of molecular weights 55 and $60 \mathrm{kDa}$, whereas cultures receiving four doses had increased $\varepsilon(\gamma-$ glutamyl)lysine present in a protein of around $75 \mathrm{kDa}$. The crosslink was also present in high-molecular-weight protein polymers, which were unable to enter the stacking and resolving gels.

\section{TG2 treatment affects both matrix deposition and turnover}

To establish whether TG2-mediated crosslinking affects total ECM protein or collagen deposition and turnover, angiogenesis cultures were pulse-labeled with $\left[2,3-{ }^{3} \mathrm{H}\right]$ proline and [2,3- $\left.{ }^{3} \mathrm{H}\right]$ amino-acid mixture, respectively (Figure $4 \mathrm{c}$ ). Following the removal of the pulse from the media, the amount of the radiolabel present in the matrix was then measured. Untreated cultures demonstrated a time-dependent decline in amount of radioactivity present in the ECM. In contrast, TG2 treatment led to a significant $(P<0.05)$ increase in the amount of radiolabel found in the ECM, indicating that the application of the enzyme initially increases both collagen and total matrix deposition, and then prevents its turnover, since the amount of radiolabel retained in the ECM remained relatively stable with time.

\section{TG2 promotes collagen I fibrillogenesis in vitro}

Addition of TG2 to neutralized type I collagen in vitro increased the rate of fibril formation, as measured by turbidity in a dose-dependent manner (Figure 5a). Interestingly, a decrease in the final level of fibril formation was noted with TG2 treatment. Inhibition of transglutaminase activity using the TG2 site-directed inhibitor NTU281 (500 $\mu \mathrm{M})$ confirmed that these effects were solely due to TG activity.

Figure 2 Exogenous TG2 treatment inhibits angiogenesis in vitro. (a) shows the dose-dependent effect of TG2 treatment on capillary development in the human angiogenesis coculture system. The top left panel shows capillary development in untreated cultures. The top right panel shows cultures receiving two TG2 treatments $(25 \mu \mathrm{g} / 0.5 \mathrm{ml}, 50 \mathrm{U}$ per treatment) on days 2 and 5 . Bottom left, cultures treated four times with TG2 on days 2, 5, 7, and 10. Bottom right, prior inactivation of the applied TG2 by inhibitor NTU283 neutralizes the antiangiogenic effect of four TG2 doses. HUVEC were stained using an anti-vWF antibody, original magnification is $\times 8$. (b) shows quantification of the vWF staining in (a) by image analysis. The mean number of vessel branch points, percentage area covered by vessels, vessel number, and vessel length were the capillary characteristics analyzed following two (gray bars), four (black bars), or four inactive (hatched bars) TG2 treatments compared to controls (open bars). Data shown are the mean values obtained from analysis of 12 nonoverlapping fields of view from a representative experiment; bars, \pm S.D. *Significant difference compared to control $(P<0.05)$. (c) TG2 treatment inhibits angiogenesis in a rat aorta segment model of angiogenesis. Aorta segments were obtained from 10weeks-old rats and implanted into Matrigel and cultured for up to 14 days. The top left is a phase-contrast image demonstrating endothelial cell outgrowth after 8 days in culture (original magnification $\times 2$ ). The top middle panel shows staining of tubules for vWF following 14 days in culture, the arrow indicates the position of the aortic explant (original magnification $\times 4$ ). The top right (original magnification $\times 8$ ) and bottom left (original magnification $\times 40$ ) panels demonstrate capillary formation in untreated cultures (arrows). The bottom middle (original magnification $\times 40$ ) and bottom right (original magnification $\times 40$ ) panels show inhibition of capillary formation in cultures receiving four daily TG2 treatments $(50 \mu \mathrm{g} / \mathrm{ml}, 50 \mathrm{U}$ per treatment) once outwardly migrating endothelial cells were detected (day 8 or 9$)$ 

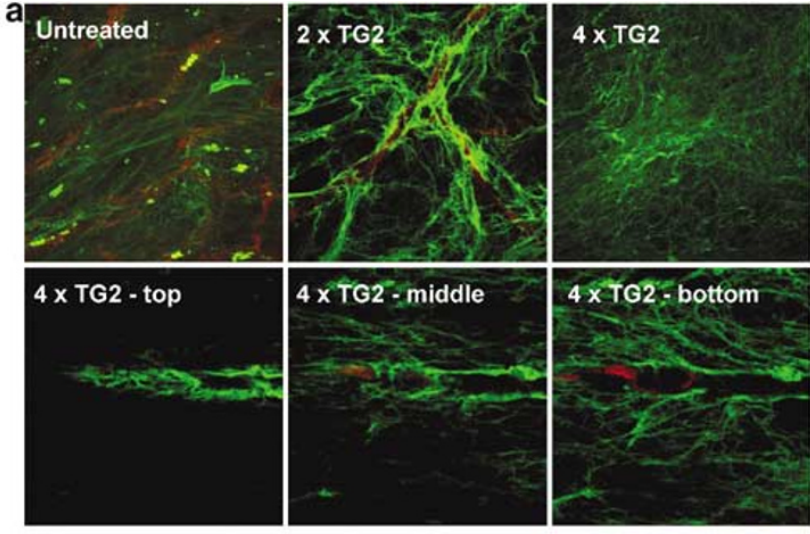

b

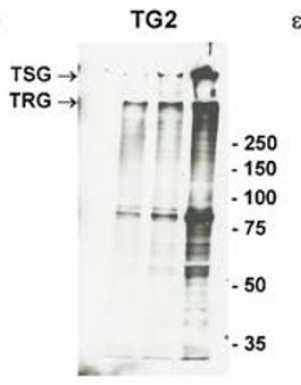

$\varepsilon(\gamma$-glutamyl)lysine

fibronectin
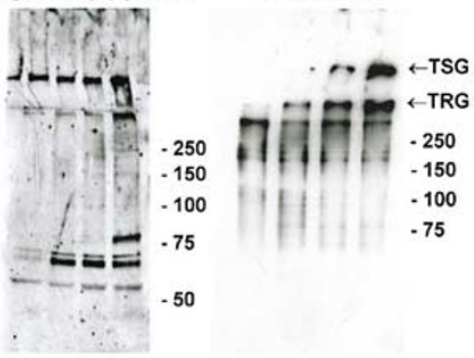

$-250$

.100

.75
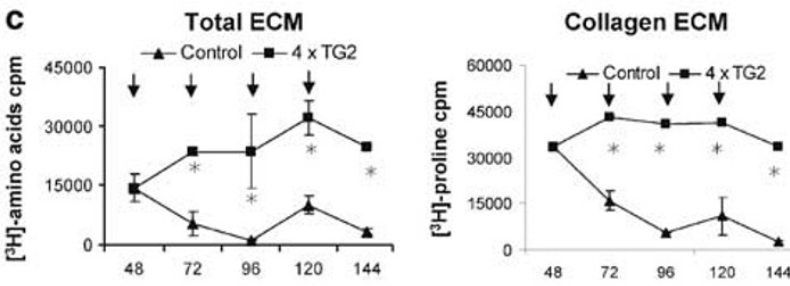

Figure 4 TG2 applied to angiogenesis cultures forms an artificial, crosslinked ECM that is resistant to turnover. (a) shows distribution of in situ TG2 activity (fluorescein cadaverine incorporation - green) and HUVEC (vWF staining - red) in TG2-treated cultures. Photomultiplier settings were optimized to show detail in ECM structure and not reflect differences in fluorescence intensity. The top left panel shows untreated cultures (original magnification $\times 40$ ). The top middle panel shows cells receiving two TG2 treatments on days 2 and 5 . TG2 activity is seen in thick fibrillar structures that coat the capillaries and radiate into the surrounding fibroblast layer (original magnification $\times 40$ ). The top right panel shows TG2 activity in cells treated with four doses of enzyme on days 2, 5, 7, and 10. The TG2 matrix becomes a course mesh and HUVEC are largely absent (original magnification $\times 40$ ). The bottom panels show higher magnification images (original magnification $\times 63$ ) of in situ activity and HUVEC in sequential confocal sections through the top (bottom left), center (bottom middle) and base (bottom right) of a capillary treated four times with TG2. (b) shows nonreducible TG2-mediated protein polymerization in enzyme-treated cultures by Western blotting. Left panel, immunoblot analysis with anti-TG2 antibody; middle panel, immunoblotting of $\varepsilon(\gamma$-glutamyl)lysine; right panel, immunoblotting of fibronectin. Lanes: 1, untreated cultures; 2, one TG2 treatment; 3, two doses of TG2; 4, four TG2 treatments. TSG indicates the top of the stacking gel; TRG shows the top of the resolving gel, molecular weights $(\mathrm{kDa})$ are indicated to the right of blots. $(\mathbf{c})$ shows the effect of TG2 treatment on ECM deposition and turnover in a coculture angiogenesis model. Total ECM deposition and turnover is shown left, collagen deposition and turnover is shown right. Pulse labeling of angiogenesis cultures with $\left[2,3-{ }^{3} \mathrm{H}\right]$ proline and $\left[2,3{ }^{3} \mathrm{H}\right] \mathrm{amino}$ acids was undertaken as described in Materials and methods; data show radioactivity present in ECM following TG2 treatment after pulse labeling. The arrows indicate time points for TG2 addition. Data show mean values $(n=4)$ from a representative experiment; bars, \pm S.D. *denotes significant difference compared to control $(P<0.05)$
TG2-mediated crosslinking of a cell-deposited matrix confers resistance to degradation by matrix metalloproteinase (MMP)-1

To assess whether TG2-mediated crosslinking confers increased proteolytic resistance to a cell deposited matrix, C378 cultures were pulse-labeled with $\left[2,3-{ }^{3} \mathrm{H}\right.$-proline], treated with exogenous active TG2, and, once the cells were removed by treatment with sodium deoxycholate, the remaining ECM was subsequently exposed to preactivated proMMP-1 for $16 \mathrm{~h}$. The susceptibility of the matrix to active MMP-1 was measured as a percentage of total collagen digestion (Figure 5b). Untreated cultures demonstrated increased susceptibility to MMP-1, with approximately $50 \%$ of the total collagen matrix being digested when exposed to the MMP. In contrast, in the TG2-treated cultures, only around $25 \%$ of the extracellular collagen matrix was broken down and released under the same conditions.

\section{TG2 downregulates collagen I, III, and IV expression}

Given that collagen deposition into the ECM of the angiogenesis cultures increased following TG2 treatment, we next explored the possibility that TG2 may upregulate collagen expression, possibly through TGF- $\beta$ signaling. Paradoxically, addition of exogenous TG2 administration (four daily doses of $50 \mu \mathrm{g} / \mathrm{ml} ; 50 \mathrm{U} / \mathrm{ml})$ to $\mathrm{C} 378$ cultures was found to correlate with a significant $(P<0.05)$ decrease in collagen I, III, and IV expression when analyzed by Northern blotting (Figure 5c).

\section{TG2 induced matrix deposition inhibits tumor growth in vivo}

As a continuum to the in vitro experiments with exogenous TG2, the ability of this enzyme to modulate matrix deposition leading to inhibition of tumor growth was also examined. Mice bearing tumors of CT26 colon carcinoma cells received five separate $50 \mu \mathrm{l}$ intratumor injections of either TG2 $(4 \mathrm{mg} / \mathrm{ml}$, $400 \mathrm{U} /$ injection containing $2 \mathrm{mM} \mathrm{Ca}^{2+}$ and $3 \mathrm{mM}$ dithiothreitol (DTT)) or PBS (containing $2 \mathrm{mM} \mathrm{Ca}^{2+}$ and $3 \mathrm{mM} \mathrm{DTT}$ ). TG2 treatment delayed tumor growth, and led to increased animal survival (Figure 6a) when compared to controls. Following TG2 treatment, $20 \%$ of animals demonstrated complete tumor regression, with the rest showing a delay in tumor growth. Staining of recovered tumor sections for TG2 antigen confirmed deposition of the applied enzyme within the body of the tumor, whereas control tumors revealed endogenous TG2 staining only in the tumor vasculature (Figure 6b). As expected from the in vitro studies, no organized vascular structures could be detected in TG2-treated tumors when stained for vWF. Tumors administered with four applications of TG2 exhibited diffuse VWF staining, indicative of capillary structures in disarray. In contrast, complete endothelial lumen structures were observed in the PBS controls (Figure 6b). Moreover, TG2-treated tumors presented collagen I staining in fibrotic-like areas that were absent from PBS-treated animals (Figure 6b). In addition, Masson's trichrome staining revealed augmented deposition of collagen fibers in TG2treated tumors that appeared to seal off the tumor body. In 

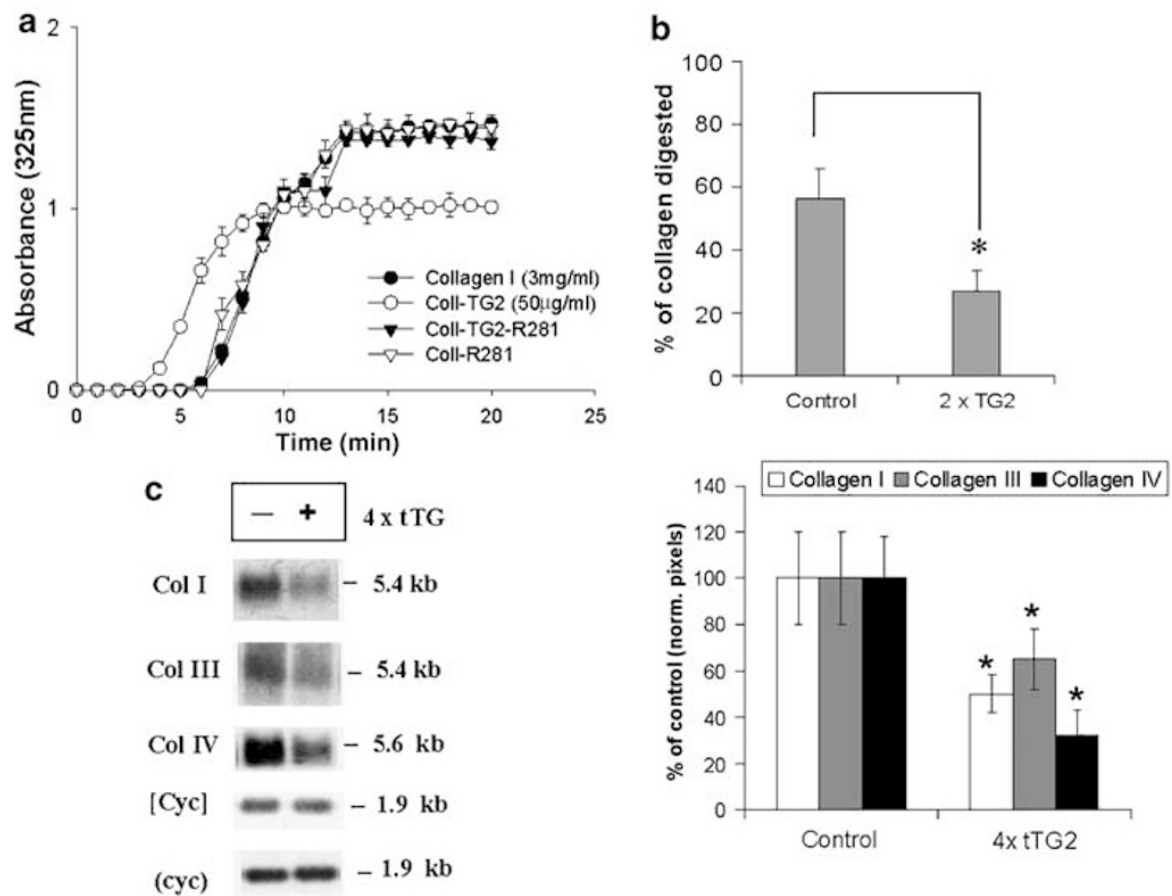

Figure 5 TG2 promotes in vitro collagen fibrillogenesis and ECM resistance to MMP-1 degradation, while downregulating collagen expression. (a) shows that TG2 stimulates collagen fibril formation $(\bigcirc)$ compared to untreated collagen $(\bullet)$, collagen treated with NTU281-inactivated TG2 $(\mathbf{\Delta})$ or collagen with NTU281 alone $(\triangle)$. Results represent the mean from three independent experiments; bars, \pm S.D. In (b), TG2 is shown as conferring resistance to ECM degradation by MMP-1. TG2treated C378 dermal fibroblasts previously labeled with [2,3- $\left.{ }^{3} \mathrm{H}\right]$ proline were stripped of cells and the ECM exposed to active MMP-1 for $16 \mathrm{~h}(P<0.05)$. Values represent mean \pm S.D. $(n=6)$ from two separate experiments; ${ }^{*}$ indicates significant difference compared to control $(P<0.05)$. (c) shows that TG2 downregulates expression of collagen I, III, and IV mRNA in treated C378 dermal fibroblasts. Cyclophillin probing (Cyc) was used for collagen I and IV normalization, whereas [cyc] was used for collagen III. Densitometric values represent mean \pm S.D. $(n=3)$; *significant difference compared to control $(P<0.05)$

contrast, collagen staining was largely absent in PBS-treated tumors (Figure 6b). Analysis of tumors for the TG2-mediated $\varepsilon(\gamma$-glutamyl)lysine iso-dipeptide and hydroxyproline, as a measure of collagen, demonstrated significant $(P<0.05)$ increases over PBS-treated tumors (Figure 6c).

\section{Tumor growth in $\mathrm{TG}^{-l-}$ and $\mathrm{TG2}^{+/+}$mice}

As a further means of demonstrating the importance of TG2 in arresting tumor growth, C57BL/6 mice lacking TG2 (TG2 ${ }^{-{ }^{-}}$) and their controls (TG2 ${ }^{+/+}$) were implanted subcutaneously with the compatible tumor B16F1 (Figure 7a). Tumors implanted into the $\mathrm{TG}_{2}{ }^{-I-}$ mice showed a significant increase in tumor growth between 16 and 22 days compared to TG2 $2^{+/+}$ mice, resulting in an decrease in the number of animals surviving by up to 8 days (Figure 7a). Staining of tumor sections with mouse anti-TG2 antibody revealed the presence of TG2 antigen in the wild-type mice, which appeared to be mainly confined within the host tissue immediately surrounding the tumor. TG2 expression was virtually absent within the tumor body of both wild-type and knockout mice, as indicated by immunofluorescent staining (Figure 7b) and Western blotting (Figure 7c).

\section{Discussion}

Our initial focus in this investigation was to assess the importance of TG2 in HUVEC undergoing angiogenesis. It has been suggested that TG2-mediated ECM crosslinking in HUVEC and the endothelial-like cell line ECV304 contributes to basement membrane assembly and cell adhesion. ${ }^{8,12}$ We found abundant, endogenous TG2 in capillaries grown in vitro (Figure 1), with enzyme activity localized in the HUVEC ECM. Inhibition of the endogenous TG2 had little effect on tube formation, suggesting that the observed in situ extracellular activity of TG2 is not essential for tube formation. This is in keeping with the observation that TG2 knockout mice have not so far been reported to show any vascular abnormalities. ${ }^{14}$

We next explored whether increasing the amount of TG2 found in the extracellular environment could affect capillary tube formation. Sequential additions of active TG2 to two separate angiogenesis models, a coculture system containing HUVEC and dermal fibroblasts and a well-established aorta segment model, led to a nontoxic, dose-dependent angiogenesis blockade, with an optimum inhibitory dose of four additions of TG2 at a concentration of $50 \mu \mathrm{g} / \mathrm{ml}$ (Figures 2 and 3). We have previously shown that this concentration of enzyme is capable of causing matrix changes when added to dermal fibroblasts. ${ }^{19}$ Detailed analysis of the deposited ECM in the coculture model indicated that the applied TG2 led to the accumulation of an intricate matrix that surrounded capillaries, resulting in the blockade of capillary growth. It is important to note that this level of extracellular TG2 is unlikely to be present in any normal physiological situation, and represents the amount of TG2 required to form an artificial ECM that coats the culture. However, it is also difficult to gauge the 
a

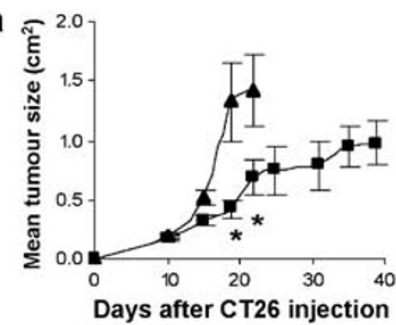

b
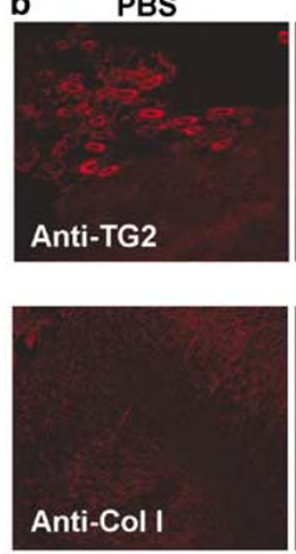

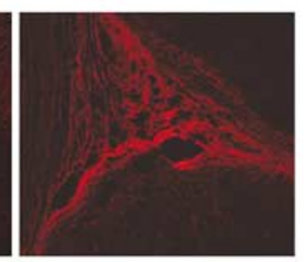

C

$4 \times$ TG2

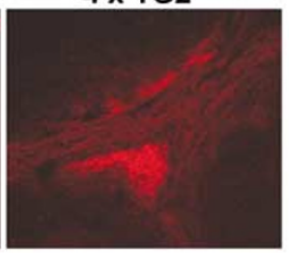

$\varepsilon(\gamma$-glutamyl)lysine

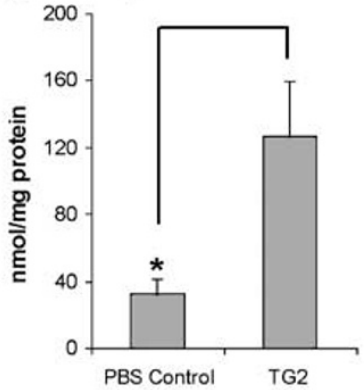

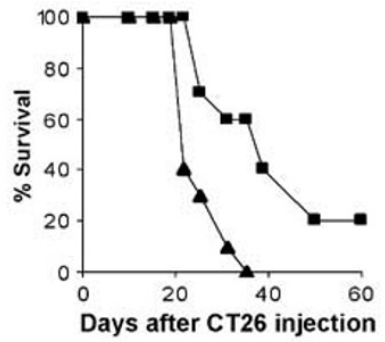

PBS
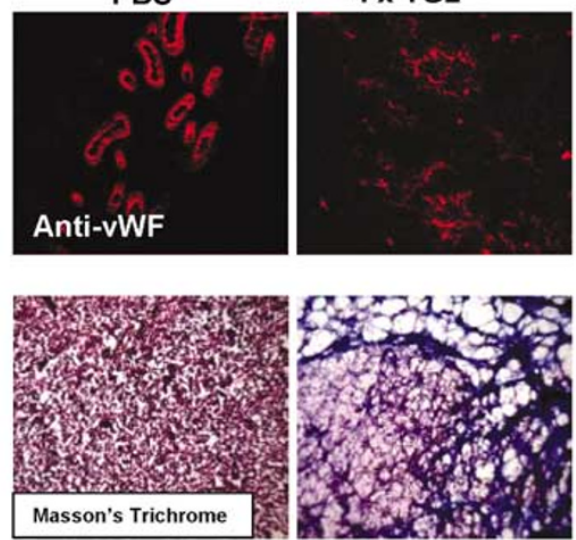

Hydroxyproline

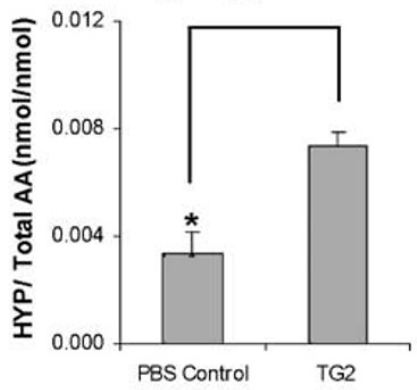

Figure 6 TG2 inhibits tumor growth and stimulates tumor fibrosis. (a) Mean tumor sizes (left panel) produced by CT26 cells in Balb/c mice following TG2 ( $\mathbf{\square}, n=10$ ) or PBS $(\boldsymbol{\Lambda}, n=10)$ treatment. Tumors received $5 \times 50 \mu$ linjections of TG2 (200 $\mu \mathrm{g} \mathrm{TG2} / 400$ U per injection) or PBS on days 10, 12, 14, 16, and 18 after initial tumor cell implantation. Data show a representative experiment from six trials; bars show + S.E. *Significant difference compared to control $(P<0.05)$. The right panel shows \% survival of Balb/c hosts following TG2 or PBS tumor treatment. (b) Immunohistochemistry and Masson's trichrome staining of recovered CT26 tumors. The top left panels show that TG2 staining is only detected in blood vessels in PBS-treated tumors, whereas large TG2 deposits are seen near fibrotic regions in TG2-treated tumors (original magnification $\times 10$ ). The top right panels show blood vessels (anti-vWF) in PBS-treated tumors, which are not seen in TG2-treated tumors (original magnification $\times 40$ ). The bottom left panels show collagen I staining in PBS-treated tumors, which is increased in TG2-treated tumors (original magnification $\times 10$ ). The bottom right panels show Masson's trichrome staining of tumors; networks of collagen fibers are abundant in TG2-treated tumors (dark blue) but absent in PBS-treated tumors (cells are pink, original magnification $\times 10)$. (c) demonstrates that $\varepsilon(\gamma$-glutamyl)lysine isopeptide (left panel) and hydroxyproline (right panel) are significantly increased $\left({ }^{*} P<0.05\right)$ in recovered TG2-treated tumors compared to PBS-treated tumors $(n=4)$

concentration of active TG2 once added to the culture medium, since much of it is likely to be sequestered by the fibronectin-containing serum. Perhaps the closest physiological equivalent is in situations of chronic tissue damage, where increased extracellular TG2 both secreted and released by virtue of cell damage leads to increased crosslinking, promoting massive tissue scarring and fibrosis. $9,10,17,19,20$ In the present study, the TG2-crosslinked ECM is likely to be composed of TG2 autopolymers, together with heteropolymers of TG2, fibronectin, or other ECM proteins deposited by the coculture system, as shown by Western blotting.
Previous work has shown contradictory results for the effects of TG2 on angiogenesis. ${ }^{17,18}$ In a window flap model, topical application of $40 \mu \mathrm{g} / \mathrm{ml}$ TG2 postsurgery was found to inhibit both growth of new vasculature and tumor implantation, when a solid piece of the R3230Ac tumor was added postsurgery to the wound. ${ }^{18}$ In contrast, the same authors demonstrated using a similar window flap model, but without tumor implantation, that three separate doses of active TG2 added on days 0,1 , and 2 postsurgery led to a stimulation of angiogenesis. ${ }^{17}$ It is difficult at this stage, given the number of variables present in the two experiments, to reconcile these contradictory findings. In both reports, no activity data were 
a

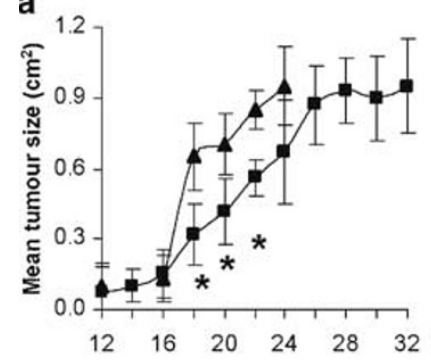

Days after B16F1 injection

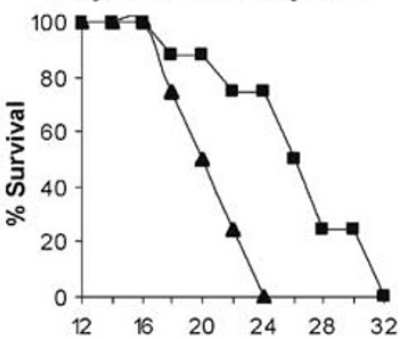

b
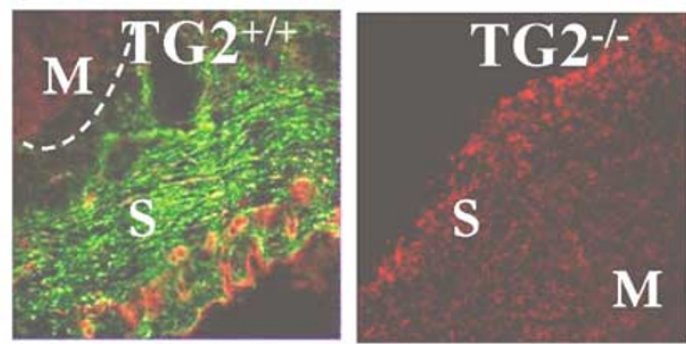

TG2
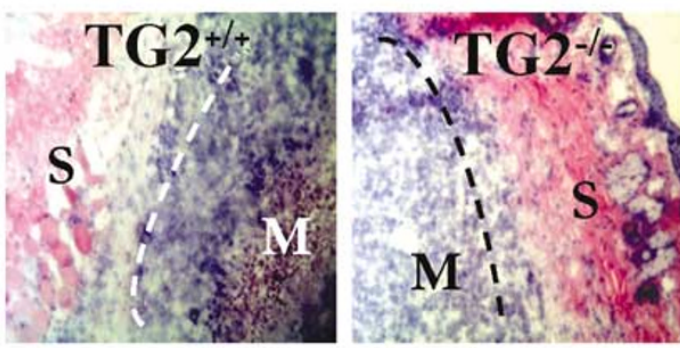

C

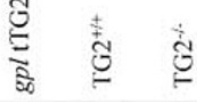

$75 \mathrm{kD} \rightarrow$

Figure $7 \mathrm{~B} 16 \mathrm{~F} 1$ melanoma tumor progression is more rapid in $\mathrm{TG}^{-l-}$ mice than wild-type $\mathrm{TG}^{+/+}$controls. (a) Representative experiment showing the mean tumor size (left panel) in challenged animals (TG2 ${ }^{-1-}$ mice, $\mathbf{\Delta} ; \mathrm{TG} 2^{+l+}$ mice, $\mathbf{\square} ; n=8$ ) and \% host survival (right panel). \% survival was monitored from day 8 onwards when differences could be detected. Data are mean values $(n=8) \pm$ S.D. * denotes significant difference compared to control $(P<0.05)$. (b) Immunofluorescent TG2 staining (mouse Cub7402 Mab) of B16-F1 tumors developing in TG2 ${ }^{-I-}$ (upper left panel) and TG2 ${ }^{+I+}$ (upper right panel) mice, revealed with anti-mouse-FITC conjugate. Green fluorescence indicates TG2 antigen, whereas nuclei counterstained with propidium iodide are shown in red $(n=4)$. Lower panels demonstrate H\&E staining of separate B16-F1 tumors developing in TG2 ${ }^{+1+}$ (lower left panel) and TG2 ${ }^{-1-}$ (lower right panel) mice. Dashed lines denote the host-neoplasm interface. S indicates surrounding host tissue, whereas M indicates melanoma tissue. (c) Western blot analysis with CUB7402 anti-TG2 Mab indicates the absence of TG2 antigen within the tumor body of TG2 ${ }^{+I^{+}}$and TG2 ${ }^{-l-}$ B16-F1 tumors. Lane 1, $100 \mathrm{ng}$ gpl TG2; lane 2, TG ${ }^{+I^{+}}$tumor; lane $3, \mathrm{TG}^{-I_{-}}$tumor $(n=3)$

given for the applied enzyme, even though it was demonstrated at least in the open tumor model ${ }^{18}$ that transamidating activity was essential for the observed effects.

Given the contradictory results between these previous studies and ours, it was essential to clarify the biochemical/ cellular mechanisms whereby TG2-mediated crosslinking blocked angiogenesis in the two angiogenesis models used in our studies. By pulse-labeling cocultures with either $\left[{ }^{3} \mathrm{H}\right]$ proline or $\left[{ }^{3} \mathrm{H}\right]$ amino acids and observing radiolabel in the ECM, we monitored the effect of TG2 on both collagen and total matrix deposition and turnover in treated cultures, respectively (Figure 4). Following a single addition of TG2, both collagen and total matrix deposition were initially increased and the amount of radiolabel present in the matrix then remained relatively stable, following subsequent TG2 treatments. In contrast, in untreated cultures the radiolabel rapidly decreased as the matrix was proteolytically degraded. In previous studies, we have demonstrated that in vitro crosslinking of collagen and collagen/fibronectin mixtures leads to an increased resistance to their breakdown by MMP$1 .^{21}$ In this report, we further demonstrate that a conditioned, cell-deposited matrix laid down by TG2-treated dermal fibroblasts becomes more resistant to degradation by MMP-1 (Figure 5). Results from this experiment support our previous observations made in vitro ${ }^{21}$ and the results obtained from the turnover studies in that a conditioned cell-deposited matrix crosslinked by TG2 has increased resistance to MMP digestion, thus accounting for its slower rate of turnover. The added observation that TG2 activity can increase the rate of collagen fibril formation provides a further biochemical mechanism to account for the increase in collagen deposition after TG2 addition (Figure 5). Moreover, in previous reports it has also been demonstrated that overexpression of TG2 in Swiss 3T3 fibroblasts leads to the increased accumulation of both fibronectin and the latent TGF- $\beta$-1-binding protein. ${ }^{22}$

It is well established that modulation of the ECM is central to many aspects of endothelial cell behavior during angiogenesis and that collagen metabolism in particular may exert an effect on the angiogenic cascade. It has been reported that the antiangiogenic properties of proline analogs in combination with angiostatic steroids are attributed to their ability to 'switch-off' collagen accumulation. ${ }^{23}$ Paradoxically, in this study we demonstrate that ECM accumulation caused by increased protein crosslinking can lead to compromised endothelial cell tube formation. Inhibition of angiogenesis by TG2 is therefore mediated by altering the homeostasis of ECM turnover towards deposition rather than the initial destabilization of the matrix, which is a prerequisite for endothelial cell proliferation and migration. ${ }^{16}$ We have also established that cells confined in such a rigid ECM 
environment may respond by downregulating collagen I, III, and IV expression (Figure 5). This suggests that a feedback mechanism might be set into motion by the increased deposition of collagen in the ECM, in order to try and maintain ECM homeostasis. Such a change in cell behavior might occur by an outside-in signaling mechanism facilitated by the mechanochemical signaling known to occur as the ECM environment becomes increasingly rigid, due to increased crosslinking. ${ }^{16}$ The observation that collagen expression is downregulated also suggests that TG2 is not exerting its effect via activation of the protein synthesis pathway that is triggered by TGF- $\beta-1 .^{21}$

Our observations that TG2 could promote 'in vitro fibrosis', as defined by the increased deposition and stabilization of matrix proteins, leading to blockade of endothelial cell tube formation, led us to pose the question whether modulation of the ECM by TG2 could be used in vivo to inhibit tumor growth and progression (Figure 6). Many, but not all, tumors show reduced TG2 activity, ${ }^{24}$ while overexpression of TG2 in the malignant hamster fibrosarcoma cell line $\mathrm{MetB}^{25}$ and in a neuroblastoma SK-N-BE ${ }^{2}$-derived cell line ${ }^{26}$ leads to a reduced incidence of tumor development. Such observations might be attributed to the ability of the enzyme to modulate both deposition and stabilization of the ECM. It is now recognized that modulation of the ECM in the tumor microenvironment plays an important role in the regulation of tumor cell growth, metastasis, and angiogenesis. ${ }^{27}$ Generally, tumor growth and angiogenesis require a thinning of the ECM facilitated by increased matrix turnover brought about by secretion of a host of proteases. ${ }^{16}$ Evidence suggests that the host animal tries to elicit a wound response against the tumor by encapsulating the neoplasm at the tumor edges. This normally takes the form of granulation tissue at the tumor-stroma interface, which is remodeled into collagen 'scar tissue' and may, to some extent, limit tumor invasion. ${ }^{28,29}$ Our studies using TG2 null mice $\left(\mathrm{TG}^{-{ }^{--}}\right)$indicate that the B16-F1 melanoma cells grow faster in the TG2 null mice, leading to decreased animal survival when compared to the control animals that express normal levels of TG2 (TG2 ${ }^{+/+}$; Figure 5). Given that TG2 is now recognized as a wound response enzyme, and taking into account immunohistochemical evidence from this study that reveals the presence of TG2 antigen within the host tissue surrounding the tumor, it is not unreasonable to suggest that the enzyme may have a role in the host's defense mechanism. To this end, in addition to its potential role in providing a barrier to tumor spread, the enzyme has been implicated as a necessary component for the phagocytosis of apoptotic cells by macrophages. ${ }^{30}$ Hence, it cannot be fully ruled out that the increased rate of tumor growth in the $\mathrm{TG}^{-1-}$ mice is in part due to this.

Our studies demonstrating that in TG2 wild-type mice the enzyme is found upregulated in the host tissue surrounding the B16-F1 melanoma is supported by an immunohistochemical study in human mammary tumors, in which TG2 was also shown to be associated with the host's ECM surrounding the tumor. ${ }^{18}$ Our studies in vitro using the coculture model of angiogenesis and in vivo demonstrating TG2 involvement in tissue fibrosis ${ }^{9,21,31}$ indicate that TG2 can indeed regulate the balance between ECM degradation and accumulation. Moreover, preliminary studies undertaken on the R3230Ac tumor implanted into a skin flap window chamber indicated that the topical addition of TG2 could limit tumor growth with a concomitant decrease in angiogenesis. ${ }^{18}$ However, in this model the open tumor was immediately accessible for topical application of TG2, allowing even distribution of the enzyme to the tumor-host interface. In addition, this type of tumor model does not test the effects of TG2 on a tumor that is already established and growing, which is essential for any potential therapeutic application of the enzyme in a clinical setting. To test the use of the enzyme in a more applicable preclinical situation, TG2 was injected directly into growing colon carcinoma tumors, previously implanted by subcutaneous injection of CT26 cells into mice. Of those tumors injected $20 \%$ demonstrated complete tumor regression, while the rest showed a delay in tumor growth. The increased presence of both collagen and the isodipeptide crosslink ( $\varepsilon(\gamma$-glutamyl) lysine) in the tumor mass confirms that TG2 can modulate the tumor ECM, leading to a fibrotic response and a change in cell behaviour as previously observed in the in vitro angiogenesis cultures. Interestingly, in a similar context, mice lacking the urokinase plasminogen activator receptor-associated protein (UPARAP), which mediates intracellular collagen degradation, were able to contain tumor progression unlike their wildtype counterparts. ${ }^{32}$ This study confirms the importance of collagen accumulation within the tumor as a barrier to tumor invasion. In our experimental model, rather than modulating the capacity of stromal cells to digest collagen either intracellularly (lysosomes) or extracellularly (MMPs), TG2induced overloading of the tumor ECM with collagen provides a comparable barrier to tumor progression. Additionally, in this TG2-induced ECM, we could not detect regularized vasculature by vWF staining, suggesting that, as found in the in vitro angiogenesis models, TG2-mediated crosslinking can destabilize angiogenesis (Figure 6).

The findings which demonstrate that an excess of exogenous TG2 delivered in vivo can lead to fibrogenesis are consistent with the enzyme's observed pathological role in diabetic nephropathy, liver fibrosis, and hypertrophic scarring. ${ }^{9,10,33}$ Importantly, in this instance, the enzyme has been used to inhibit tumor growth by a mechanism involving increased stabilization of the ECM, leading to changes in cell behavior that limit tumor invasion and reduce angiogenesis, thus enhancing the host's defense mechanisms. While considerable advances have been made in our understanding of angiogenesis, the development of effective tumor therapeutic strategies that rely on angiogenesis blockade has been less successful when animal studies have been translated into clinical phase trials. ${ }^{4}$ Modulating cell behavior in a tumor by inducing changes in the ECM such that deposition instead of degradation is favored may provide a more rational approach for the development of new cancer therapies.

\section{Materials and Methods}

\section{Cell culture}

Human angiogenesis cell model (TCS Cellworks, Buckingham, UK), a coculture system containing HUVEC and human dermal fibroblasts ${ }^{34}$ that deposit their own ECM and that spontaneously form capillaries over an 
11-day period, were cultured in the growth medium supplied by the manufacturers. Active guinea-pig liver TG2 was initially obtained from Sigma (Dorset, UK), but was for the majority of studies purified in house as described previously. ${ }^{35}$ TG2 was applied to the cultures $(50 \mu \mathrm{g} / \mathrm{ml}, 50 \mathrm{U} /$ $\mathrm{ml}$ ) either once on day 2, twice on days 2 and 5 , or four times on days 2, 5, 8, and 10. In all, $1 \mathrm{U}$ of enzyme activity equals $1 \mathrm{nmol}$ of putrescine incorporated in $N, N^{\prime}$-dimethylcasein per hour. Cultures were also treated in parallel with TG2, inactivated by preincubation in $500 \mu \mathrm{M}$ of the specific active site-directed inhibitor NTU283 (1,3-dimethyl-2[(oxopropyl)thio] imidazolium) for $6 \mathrm{~h}$ at $4{ }^{\circ} \mathrm{C} .^{36}$ Any excess inhibitor was removed by dialysis against $5 \mathrm{mM}$ Tris- $\mathrm{HCl}, \mathrm{pH} 7.8$, and the enzyme tested for activity prior to addition to the cell cultures. In some experiments, NTU283 or the active site-directed inhibitor NTU281 (N-benzyloxycarbonyl (CBZ)-Lphenylalanyl-6-dimethylsulfonium-5-oxo-L-norleucine) was added directly to cell cultures at $1 \mathrm{mM}$ to assess the effect of endogenous transglutaminase inhibition on tube formation. ${ }^{37,38} \mathrm{C} 378$ human foreskin dermal fibroblasts (supplied by EJ Woods, University of Leeds, UK) and CT26 mouse colon carcinoma cells were cultured in Dulbecco's modified Eagle's medium (DMEM) containing 10\% (v/v) heat-inactivated fetal calf serum (FCS), $2 \mathrm{mM}$ L-glutamine, $100 \mathrm{U} / \mathrm{ml}$ penicillin, and $100 \mu \mathrm{g} / \mathrm{ml}$ streptomycin. HUVEC (TCS Cellworks) were cultured in large vein endothelial cell growth medium containing endothelial cell growth supplement (ECGS), 2\% FCS, $2 \mathrm{mM}$ L-glutamine, $100 \mathrm{U} / \mathrm{ml}$ penicillin, and $100 \mu \mathrm{g} / \mathrm{ml}$ streptomycin.

\section{Aorta ring angiogenesis model}

Aortas obtained from 10-week-old AS rats were cut into 10-mm-thick rings, embedded in Matrigel and cultured in large vein endothelial cell growth medium as described previously. ${ }^{39}$ Following a 10-day incubation, when endothelial cell outgrowth from the tissue was observed, cultures were treated with $50 \mu \mathrm{g} / \mathrm{ml} \mathrm{TG2}(50 \mathrm{U} / \mathrm{ml})$ for 4 days at daily intervals. Images of migrating endothelial cells and interconnected capillary tubes were captured using an Olympus digital camera.

\section{Immunohistochemistry}

In vitro angiogenesis cultures were fixed in ice-cold $70 \%$ ethanol, blocked with $3 \%$ bovine serum albumin (BSA) in PBS, and stained for TG2 or vWF using a mouse anti-TG2 monoclonal (Cub7402, Neomarkers, Cambridgeshire, UK) or rabbit anti-vWF polyclonal antibodies (Sigma, Dorset, UK and TCS Cellworks) diluted in blocking buffer. Staining was revealed using either anti-mouse IgG-horseradish peroxidase (HRP) (Perbio Science, Northumberland, UK) or anti-rabbit-HRP (Sigma) conjugate and developed with FastDAB substrate (Sigma). Capillary images were obtained using an Olympus digital camera and quantitative image analysis was performed using QWin software (Leica Microsystems, Milton Keynes, UK).

\section{Cell density and viability}

In situ cell viability in TG2-treated angiogenesis cultures was assessed using the differential LIVE/DEAD stain (Molecular Probes, Paisley, UK). Viable cells were distinguished from nonviable cells by labeling cultures with 3, 3'-dioctadecyloxacarbocyanine, which becomes membrane impermeable when metabolized by viable cells. Cells are then washed and counterstained with propidium iodide, which enters nonviable cells with compromised plasma membranes. Cell viability of TG2-treated HUVEC and C378 cells was also measured by membrane permeability using $\mathrm{LDH}$ release into the medium monitored by the Cytotox $96^{\mathrm{TM}}$ NonRadioactive Cytotoxicity Assay kit (Promega, Southampton, UK) accord- ing to the manufacturer's protocol. Cell density of HUVEC and C378 cells was measured using the XTT assay (Promega) according to the manufacturer's instructions.

\section{Visualization of in situ TG2 activity}

In situ TG2 activity was localized by incubating cultures with growth medium containing $0.2 \mathrm{mM}$ biotin cadaverine (Sigma). Cultures were fixed in ice-cold $70 \%$ ethanol, blocked with $3 \%$ BSA in PBS, then incubated with extravidin peroxidase diluted in blocking buffer. Incorporated biotin cadaverine was revealed using FastDAB substrate (Sigma). For fluorescent localization of TG2 activity in situ, cells were cultured in medium containing $0.5 \mathrm{mM}$ fluorescein-cadaverine as described previously. ${ }^{11,19}$ Cultures demonstrating capillary formation were fixed in methanol at $-20^{\circ} \mathrm{C}$ and counterstained for vWF using a rabbit anti-vWF polyclonal antibody (Sigma). vWF staining was revealed using anti-rabbit IgG-tetramethyl rhodamine isothiocyanate (TRITC) conjugate (DAKO, Cambridgeshire, UK). Stained cultures were mounted in $70 \%$ glycerol and viewed using a Leica TCSNT confocal microscope.

\section{Western blot analysis}

Angiogenesis cultures were directly solubilized in $150 \mu$ l of $2 \times$ SDSLaemmli sample buffer and separated on $8 \%$ polyacrylamide gels and electroblotted onto nitrocellulose membranes. Blotted membranes were blocked in Tris-buffered saline containing 5\% dried milk, then incubated overnight at $4{ }^{\circ} \mathrm{C}$ with mouse monoclonal anti-TG2 (Cub7402, Neomarkers), a rabbit polyclonal anti-fibronectin antibody (Sigma), or mouse anti$\varepsilon(\gamma$-glutamyl)lysine antibody (Abcam, Cambridgeshire, UK). Membranes were then incubated with anti-mouse IgG-HRP secondary antibody (Pierce), or anti-rabbit IgG-HRP antibody and bands were revealed using the enhanced chemiluminescence kit (ECL; Amersham, Buckinghamshire, UK).

\section{Total ECM and collagen turnover}

On day 5 , the angiogenesis model was labeled with $5 \mu \mathrm{Ci} / \mathrm{ml}\left[2,3-{ }^{3} \mathrm{H}\right]$ proline or $5 \mu \mathrm{Ci} / \mathrm{ml}\left[2,3-{ }^{3} \mathrm{H}\right]$ amino-acid mixture in the presence of fully supplemented growth medium. Following a 48-h incubation period, the medium was removed and cells were washed three times in PBS, replenished with radiolabel-free medium and then administered with 4 daily doses of $50 \mu \mathrm{g} / \mathrm{ml}$ active TG2 $(50 \mathrm{U} / \mathrm{ml})$. The amount of the incorporated label was measured in the cell growth medium, and in the ECM as described previously. ${ }^{19}$

\section{Collagen turbidity assay}

Collagen fibrillogenesis was monitored using a spectrophotometric method. Type I collagen (Sigma) was solubilized in $0.2 \mathrm{M}$ acetic acid at a concentration of $5 \mathrm{mg} / \mathrm{ml}$ at $4^{\circ} \mathrm{C}$ with constant stirring for $24 \mathrm{~h}$. Fibrillogenesis was initiated by neutralization of the collagen mixture through addition of $10 \times$ DMEM and $0.2 \mathrm{M}$ HEPES buffer to final concentrations of $1 \times$ and $0.02 \mathrm{M}$, respectively. Addition of $\mathrm{CaCl}_{2}$ and DTT to a final concentration of $5 \mathrm{mM}$ was made immediately after neutralization and prior to the addition of guinea-pig liver TG2. Neutralized collagen $(3 \mathrm{mg} / \mathrm{ml})$ was then treated with $50-250 \mu \mathrm{g} / \mathrm{ml}$ of active TG2 (50$250 \mathrm{U} / \mathrm{ml})$. Inhibition of transglutaminase activity was achieved using $500 \mu \mathrm{M}$ of active site-directed inhibitor NTU281. Absorbance was measured at $325 \mathrm{~nm}$ at a fixed temperature of $25^{\circ} \mathrm{C}$. 


\section{Collagen digestion by MMP-1 and proteinase $\mathrm{K}$}

C378 human dermal fibroblasts were seeded at $2 \times 10^{5}$ cells/well in 24well tissue culture plates and labeled the following day with $5 \mu \mathrm{Ci} / \mathrm{ml}$ $\left[2,3-{ }^{3} \mathrm{H}\right]$ proline (Amersham, Buckinghamshire, UK) for $48 \mathrm{~h}$. Following pulse-labeling, cells were treated daily and for 2 days with $50 \mu \mathrm{g} / \mathrm{ml}$ of active TG2 $(50 \mathrm{U} / \mathrm{ml})$. Cells were washed twice in PBS, and removed by exposure to $0.1 \%$ deoxycholate $/ 2 \mathrm{mM}$ ethylene diamine tetraacetic acid (EDTA) for $10 \mathrm{~min}$ at room temperature. The remaining ECM fraction was then washed twice in PBS and exposed initially to $200 \mu \mathrm{l}$ of $100 \mathrm{ng} / \mathrm{ml}$ activated pro-MMP-1 in $0.1 \mathrm{M}$ Tris- $\mathrm{HCl}, \mathrm{pH} 7.5$, and $1 \mu \mathrm{M} \mathrm{ZnCl}_{2}$ for $16 \mathrm{~h}$. Pro-MMP-1 (Merck Biosciences, Nottingham, UK) was activated in $1 \mathrm{mM}$ p-aminophenylmercuric acetate (APMA) (Merck Biosciences) and $10 \mathrm{mM}$ $\mathrm{NaOH}$ for $2 \mathrm{~h}$ at $37^{\circ} \mathrm{C}$ as described previously. Digested collagen fragments were collected and the remaining insoluble ECM then washed twice with PBS, prior to further digestion in $100 \mu \mathrm{l}$ of $200 \mu \mathrm{g} / \mathrm{ml}$ proteinase $\mathrm{K}$ (Sigma) in $50 \mathrm{mM}$ Tris- $\mathrm{HCl}, \mathrm{pH} 7.4,10 \mathrm{mM}$ EDTA, and $10 \mathrm{mM} \mathrm{NaCl}$, followed by scraping and dissolution in $50 \mu$ of $4 \%$ SDS. The incorporated label present in the digested fractions was measured by scintillation counting (Tricarb 300, Canberra Packard, Pangbourne, UK).

\section{Northern analysis}

C378 human dermal fibroblasts were seeded on $25 \mathrm{~cm}^{2}$ flasks at $5 \times 10^{4}$ cells/flask and treated daily for four consecutive days with $50 \mu \mathrm{g} / \mathrm{ml}$ (50 U/ $\mathrm{ml}$ ) of TG2. Cells were lysed in TRI Reagent (Sigma) and total RNA $(20 \mu \mathrm{g})$ was separated by denaturing agarose gel electrophoresis and transferred to Hybond $\mathrm{N}$ nylon membranes (Amersham). Specific random primed DNA probes were obtained from the following DNA sequences: rat collagen (a1)I and III, and human collagen (a1) IV. In all, $12.5 \mathrm{ng}$ of purified cDNA was random-primed with ${ }^{32} \mathrm{P}$-labeled dCTP (Amersham) using the Prime-a-Gene system (Promega) according to the manufacturer's protocol. Hybridization was performed as described previously ${ }^{33}$ and Northern blots were quantified using Phoretix 1D image analysis software (NonLinear Dynamics, Newcastle upon Tyne, UK).

\section{CT26 tumor model}

All animal procedures were carried out in accordance with the UK Home Office Animals Act (1986). Female Balb/c mice (Harlan, Oxon, UK) were implanted with $1 \times 10^{5}$ CT26 cells in $0.1 \mathrm{ml}$ DMEM by subcutaneous injection in the right flank. After 10 days, when the tumors had reached approximately $17 \mathrm{~mm}^{2}$ in size, animals were randomly divided into two groups of 10 animals. The first group received five $50 \mu \mathrm{l}$ intratumor injections of $4 \mathrm{mg} / \mathrm{ml}$ (400 U/injection), in Dulbecco's PBS, pH 7.4, supplemented with $2 \mathrm{mM} \mathrm{CaCl}_{2}$ and $3 \mathrm{mM}$ DTT for enzyme activation on days $10,12,14,16$, and 18 after tumor cell implantation. The second treatment group received five parallel injections of $2 \mathrm{mM} \mathrm{CaCl}_{2}$ and $3 \mathrm{mM}$ DTT in PBS at the same time points. Subcutaneous tumor growth was measured at 2-days intervals with calipers and animals were killed before the individual tumor diameter exceeded $1 \mathrm{~cm}^{2}$.

\section{C57BL/6 tumor model}

Transgenic TG2 knockout and wild-type mice ${ }^{14}$ were implanted with $2 \times 10^{4} \mathrm{~B} 16$ melanoma cells in $0.1 \mathrm{ml}$ DMEM by subcutaneous injection in the right flank. After 10 days, subcutaneous tumor growth was measured at 2-days intervals with a caliper.

\section{Staining of tumor specimens}

Snap-frozen CT26 or B16 melanoma tumor tissue was embedded in OCT compound (Raymond Lamb) at $-20^{\circ} \mathrm{C}$ and sectioned at $-15^{\circ} \mathrm{C}$ using a cryostat at $10 \mu \mathrm{m}$ increments. Sections were air-dried on glass slides, then fixed in ice-cold acetone for $15 \mathrm{~min}$ before being blocked with $3 \%(\mathrm{w} / \mathrm{v})$ BSA in PBS. Depending on the tumor under investigation, sections were incubated with mouse anti-TG2 (Cub 7402, Neomarkers), goat anti-vWF (Santa Cruz, Wiltshire, UK), or goat anti-collagen type I (Chemicon, Hamps, UK). Staining was revealed by incubation with anti-mouse-TRITC conjugate (Dako), anti-mouse-fluorescein isothiocyanate (FITC) conjugate (DAKO), or anti-goat IgG-TRITC conjugate (Chemicon) as appropriate. Negative controls were obtained by omission of the primary antibody and stained sections were mounted in $70 \%(\mathrm{v} / \mathrm{v})$ glycerol and viewed on a confocal microscope (TCSNT, Leica). Collagen fibers were stained using Accustain Trichrome Stain (Sigma Diagnostics) according to the manufacturer's protocol. For hematoxylin and eosin staining of OCTembedded tumor specimens, cryostat sections were fixed in 10\% (v/v) neutral buffered formalin (Sigma) at room temperature for $20 \mathrm{~s}$ prior to rinsing in tap water for $15 \mathrm{~s}$ and staining in double-strength Carazzi's hematoxylin (Sigma) for $1 \mathrm{~min}$. Sections were once again rinsed, before being stained in $1 \%(w / v)$ aqueous eosin (Sigma) for $10 \mathrm{~s}$. Sections were finally dehydrated through increasing alcohol concentrations and slides mounted in DPX (Sigma). Images were captured under a light microscope (Ziess, Herts, UK) attached to an Olympus digital camera.

\section{Crosslink analysis}

The $\varepsilon(\gamma$-glutamyl)lysine isopeptide content of tumors was measured as outlined previously. ${ }^{9,40}$ Briefly, cell homogenates were subjected to a series of sequential proteolytic digestions, freeze-dried, and crosslink analysis was carried out by anion exchange chromatography using a LKB 4151 amino-acid analyzer.

\section{Amino-acid analysis}

Tumor specimens were hydrolyzed in $6 \mathrm{M} \mathrm{HCl}$ at $100^{\circ} \mathrm{C}$ for $16 \mathrm{~h}$, and amino acids were separated following a solid-phase extraction/derivatization protocol using the EZ-Faast amino-acid analysis kit (Phenomenex, Macclesfield, UK) according to the manufacturer's instructions. The amino-acid and hydroxyproline contents of tumors were measured against internal amino-acid standards by gas chromatography using a $9890 \mathrm{~N}$ Network GC system (Agilent Technologies, Berkshire, UK). Hydroxyproline is expressed as nmol hydroxyproline/nmol total amino acids.

\section{Statistical analysis}

Differences between data sets were determined by the Mann-Whitney or Student's $t$-test using Minitab or Sigma Stats packages at a significance level of $P<0.05$.

\section{Acknowledgements}

This work has been supported in parts by grants from the EPSRC GR/ S21755/01.

\section{References}

1. Folkman J (1995) Angiogenesis in cancer, vascular, rheumatoid and other disease. Nat. Med. 1: 27-31

2. Brown PD and Giavazzi R (1995) Matrix metalloproteinase inhibition: a review of antitumor activity. Ann. Oncol. 6: 967-974 
3. Griffioen AW, Tromp SC and Hillen HF (1998) Angiogenesis modulates the tumour immune response. Int. J. Exp. Pathol. 79: 363-368

4. Eisterer W, Jiang X, Bachelot T, Pawliuk R, Abramovich C, Leboulch P, Hogge $D$ and Eaves $C$ (2002) Unfulfilled promise of endostatin in a gene therapyxenotransplant model of human acute lymphocytic leukemia. Mol. Ther. 5: 352-359

5. Korner G, Schneider DE, Purdon MA and Bjornsson TD (1989) Bovine aortic endothelial cell transglutaminase. Enzyme characterization and regulation of activity. Biochem. J. 262: 633-641

6. Lorand $L$ and Graham RM (2003) Transglutaminases: cross-linking enzymes with pleiotropic functions. Nat. Rev. Mol. Cell Biol. 4: 140-156

7. Aeschlimann D and Thomazy V (2000) Protein cross-linking and remodeling of extracellular matrices: the role of transglutaminases. Connect. Tissue Res. 4: $1-27$

8. Martinez J, Chalupowicz DG, Roush RK, Sheth A and Barsigian C (1994) Transglutaminase-mediated processing of fibronectin by endothelial cell monolayers. Biochemistry 33: 2538-2545

9. Johnson TS, Griffin M, Thomas GL, Skill J, Cox A, Yang B, Nicholas B, Birckbichler PJ, Muchaneta-Kubara C and Meguid El Nahas A (1997) The role of tansglutaminase in the rat subtotal nephroctomy model of renal fibrosis. J. Clin. Invest. 99: 2950-2960

10. Grenard P, Bresson-Hadni S, El Alaoui S, Chevallier M, Vuitton DA and RicardBlum S (2001) Transglutaminase-mediated cross-linking is involved in the stabilization of extracellular matrix in human liver fibrosis. J. Hepatol. 35: 367375

11. Balklava Z, Verderio E, Collighan R, Gross S, Adams J and Griffin M (2002) Analysis of tissue transglutaminase function in the migration of swiss $3 T 3$ fibroblasts. The active-state conformation of the enzyme does not affect cell motility but is important for its secretion. J. Biol. Chem. 277: $16567-16575$

12. Jones RA, Nicholas B, Mian S, Davies PJ and Griffin M (1997) Reduced expression of tissue transglutaminase in a human endothelial cell line leads to changes in cell spreading, cell adhesion and reduced polymerisation of fibronectin. J. Cell Sci. 110: 2461-2472

13. Verderio EA, Telci D, Okoye A, Melino G and Griffin M (2003) A novel RGDindependent cell adhesion pathway mediated by fibronectin-bound tissue transglutaminase rescues cells from anoikis. J. Biol. Chem. 278: 42604-42614

14. De Laurenzi $V$ and Melino $G$ (2001) Gene disruption of tissue transglutaminase. Mol. Cell. Biol. 21: 148-155

15. Bell SE, Mavila A, Salazar R, Bayless KJ, Kanagala S, Maxwell SA and Davis GE (2001) Differential gene expression during capillary morphogenesis in 3D collagen matrices: regulated expression of genes involved in basement membrane matrix assembly, cell cycle progression, cellular differentiation and G-protein signaling. J. Cell Sci. 114: 2755-2773

16. Ingber DE (2002) Mechanical signaling and the cellular response to extracellular matrix in angiogenesis and cardiovascular physiology. Circ. Res. 91: 877-887

17. Haroon Z, Hettasch JM, Lai TS, Dewhirst M and Greenberg CS (1999) Tissue transglutaminase is expressed, active, and directly involved in rat dermal wound healing and angiogenesis. FASEB J. 13: 1787-1795

18. Haroon ZA, Lai TS, Hettasch JM, Lindberg RA, Dewhirst MW and Greenberg CS (1999) Tissue transglutaminase is expressed as a host response to tumor invasion and inhibits tumor growth. Lab. Invest. 79: 1679-1686

19. Gross SR, Balklava Z and Griffin M (2003) Importance of tissue transglutaminase in repair of extracellular matrices and cell death of dermal fibroblasts after exposure to a solarium ultraviolet A source. J. Invest. Dermatol. 121: $412-423$

20. Nicholas B, Smethurst P, Verderio E, Jones RA and Griffin M (2003) Crosslinking of cellular proteins by tissue transglutaminase during necrotic cell death: a mechanism for maintaining tissue integrity. Biochem. J. 371: 413-422

21. Johnson TS, Skill NJ, El Nahas AM, Oldroyd SD, Thomas GL, Douthwaite JA, Haylor JL and Griffin M (1999) Transglutaminase transcription and antigen translocation in experimental renal scarring. J. Am. Soc. Nephrol. 10: 21462157

22. Verderio E, Gaudry C, Gross S, Smith C, Downes S and Griffin M (1999) Regulation of cell surface tissue transglutaminase: effects on matrix storage of latent transforming growth factor-beta binding protein-1. J. Histochem. Cytochem. 47: 1417-1432

23. Ingber $D$ and Folkman J (1988) Inhibition of angiogenesis through modulation of collagen metabolism. Lab. Invest. 59: 44-51

24. Keogh J, Zirvi KA, Vossough S, Slomiany A and Slomiany BL (1993) Pharmacological alterations of cellular transglutaminase activity and invasiveness in human colorectal carcinoma cells. Cancer Biochem. Biophys. 13: 209-220

25. Johnson TS, Knight CR, el-Alaoui S, Mian S, Rees RC, Gentile V, Davies PJ and Griffin M (1994) Transfection of tissue transglutaminase into a highly malignant hamster fibrosarcoma leads to a reduced incidence of primary tumor growth. Oncogene 9: 2935-2942

26. Piacentini M, Piredda L, Starace DT, Annicchiarico-Petruzzelli M, Mattei M, Oliverio S, Farrace MG and Melino G (1996) Differential growth of N- and Stype human neuroblastoma cells xenografted into scid mice: correlation with apoptosis. J. Pathol. 180: 415-422

27. Dano K, Romer J, Nielsen BS, Bjorn S, Pyke C, Rygaard J and Lund LR (1999) Cancer invasion and tissue remodeling - cooperation of protease systems and cell types. APMIS 107: 120-127

28. Dvorak HF (1986) Tumors: wounds that do not heal. Similarities between tumor stroma generation and wound healing. N. Engl. J. Med. 315: 1650-1659

29. Mazooz G, Mehlaman T, Lai T-S, Greenberg CS, Dewhirst MW and Neeman M (2005) Development of magnetic resonance imaging contrast material for in vivo mapping of tissue transglutaminase activity. Cancer Res. 65: 1369-1375

30. Szondy Z, Sarang Z, Molnar P, Nemeth T, Piacentini M, Mastroberardino PG, Falasca L, Aeschlimann D, Kovacs J, Kiss I, Szegezdi E, Lakos G, Rajnavolgyi E, Birckbichler PJ, Melino G and Fesus L (2003) Transglutaminase 2-/ - mice reveal a phagocytosis-associated crosstalk between macrophages and apoptotic cells. Proc. Natl. Acad. Sci. USA 100: 7812-7817

31. Skill NJ, Griffin M, El Nahas AM, Sanai T, Haylor JL, Fisher M, Jamie MF, Mould NN and Johnson TS (2001) Increases in renal $\varepsilon(\gamma$-glutamyl)-lysine cross-links result from compartment-specific changes in tissue transglutaminase in early experimental diabetic nephropathy: pathologic implications. Lab. Invest. 81: 705-716

32. LeBrasseur N (2005) Tumours feed on collagen. J. Cell Biol. 169: 835

33. Johnson TS, Haylor JL, Thomas GL, Fisher M and EI Nahas AM (2002) Matrix metalloproteinases and their inhibitions in experimental renal scarring. Exp. Nephrol. 10: 182-195

34. Bishop ET, Bell GT, Bloor S, Broom IJ, Hendry NF and Wheatley DN (1999) An in vitro model of angiogenesis: basic features. Angiogenesis 3: 335-344

35. Leblanc A, Day N, Menard A and Keillor JW (1999) Protein guinea pig liver transglutaminase: a modified purification procedure affording enzyme with superior activity in greater yield. Expr. Purif. 17: 89-95

36. Freund KF, Doshi KP, Gaul SL, Claremon DA, Remy DC, Baldwin JJ, Pitzenberger SM and Stern AM (1994) Transglutaminase inhibition by 2-[(2oxopropyl)thio]imidazolium derivatives: mechanism of factor XIIla inactivation. Biochemistry 33: 10109-10119

37. Griffin M, Coutts IG and Saint RE (2004) Novel Compounds and Methods of Using the Same. International Publication No. WO2004/113363 A2

38. Baumgartner W, Golenhofen N, Weth A, Hiiragi T, Saint R, Griffin M and Drenckhahn D (2004) Role of transglutaminase 1 in stabilisation of intercellular junctions of the vascular endothelium. Histochem. Cell Biol. 122: 17-25

39. Mori M, Sadahira Y, Kawasaki S, Hayashi T, Notohara K and Awai M (1988) Capillary growth from reversed rat aortic segments cultured in collagen gel. Acta Pathol. JPN 38: 1503-1512

40. Knight CR, Rees RC and Griffin M (1991) Apoptosis: a potential role for cytosolic transglutaminase and its importance in tumour progression. Biochim. Biophys. Acta 1096: 312-318 